$11-2019$

\title{
Kangaroo mother care: Opportunities and implications for rural Pakistan
}

Hasan Nawaz Tahir

Aga Khan University, hasan.nawaz@aku.edu

Zafar Fatmi

Aga Khan University, zafar.fatmi@aku.edu

Follow this and additional works at: https://ecommons.aku.edu/pakistan_fhs_mc_chs_chs

Part of the Community Health and Preventive Medicine Commons, and the Congenital, Hereditary, and Neonatal Diseases and Abnormalities Commons

\section{Recommended Citation}

Tahir, H. N., Fatmi, Z. (2019). Kangaroo mother care: Opportunities and implications for rural Pakistan. JPMA. The Journal of the Pakistan Medical Association, 69(11), 1725-1729.

Available at: https://ecommons.aku.edu/pakistan_fhs_mc_chs_chs/754 


\title{
SHORT COMMUNICATION
}

\section{Kangaroo mother care: opportunities and implications for rural Pakistan}

\author{
Hasan Nawaz Tahir, Zafar Fatmi
}

\begin{abstract}
Neonatal mortality comprises $40 \%$ of total under- 5 mortality, globally. Kangaroo mother care (KMC) is one of the most cost-effective interventions to reduce neonatal mortality. KMC does not require highend equipment, intensive care facilities or technical knowledge. A recent meta-analysis reported that KMC may reduce neonatal mortality in preterm and low birth weight neonates up to $36 \%$. A review of enablers and barriers of KMC suggests that KMC can be integrated in maternal health care system by giving awareness, involving family and giving ownership of the intervention to the community. If supported with minimal incentives it would reduce the cost of health care substantially, reduce patient burden on hospitals by reducing hospital stay in postnatal period. It will reduce financial burden, time strain and help eliminate social taboos regarding preterm and low birth weight neonates. Hospital and community based KMC interventions should be tested in Pakistan.
\end{abstract} Keywords: Kangaroo Mother Care, Kangaroo Mother Care Method, skin to skin contact, preterm, low birth weight and neonate. doi: 10.5455/JPMA.263772.

\section{Introduction}

Globally 6.3 million children under the age of five succumb to death each year and $40 \%$ of these deaths occur in the neonatal period. ${ }^{1}$ Systematic analysis of the global data between 2000-2010 found that preterm birth complications (14.1\%; 1.078 million), intrapartum-related complications $(9.4 \% ; 0.717$ million), and sepsis or meningitis ( $5.2 \% ; 0.393$ million) were the leading causes of neonatal death globally. In post-neonatal children, pneumonia (14.1\%; 1.071 million), diarrhoea (9.9\%; 0.751 million), and malaria (7.4\%; 0.564 million) claim the most lives. ${ }^{2}$ Till date Sub-Saharan Africa and South Asia have the highest rates of maternal, neonatal, and child deaths compared to any other region in the world. 3

Pakistan has a neonatal mortality rate of $42 / 1000$ live births, the number of neonatal deaths is 193,718 and $49 \%$ Department of Community Health Sciences, Aga Khan University, Karachi. Correspondence: Hasan Nawaz Tahir, Email: hasan.nawaz@aku.edu of the under 5 mortality occurs in 0-28 days of life. ${ }^{4}$ The under 5 mortality was highest in Baluchistan, followed by Punjab, Sindh, Gilgit-Baltistan, Khyber Pakhtunkhwa and Islamabad, respectively. The major causes of neonatal deaths in Pakistan are prematurity (36\%), birth asphyxia and birth trauma (23\%) and sepsis (20\%). 5

In Pakistan, between the years 2000-2010, neonatal mortality rates declined at $0.9 \%$ per year. Nonetheless, it was less than the global average reduction of $2.1 \%$ per year. Nationally there had been little focus on newborn health until the year 2000 when considerable policy changes occurred. Integration of newborn care into existing community-based maternal and child packages was done including the lady health worker programme. Since 2005, donor funding mentioning the term 'newborn' was increased more for Pakistan than for other countries in the region. 6 Despite all these efforts neonatal mortality in Pakistan has the third highest rate of neonatal mortality globally and comparatively higher than the regional countries. $^{7}$

Kangaroo Mother Care (KMC) is an evidence-based intervention that is both life-saving and cost effective. Evidence strongly support improved health outcomes among preterm or low birth weight infants as it reduces the risk of neonatal mortality by $36 \%$ compared to conventional care. The risk of sepsis, hypoglycaemia, and hypothermia decreases. ${ }^{8} \mathrm{KMC}$ follows four components which include early, continuous, and prolonged skin-toskin contact between infant and caregiver; exclusive breastfeeding; early discharge from hospital; and adequate support for caregiver and infant at home. ${ }^{8}$ Recently, World Health Organization have recommended that KMC should be the routine care for neonates weighing less than 2000 grams. ${ }^{9}$

The reasons for non-integration of KMC into health systems have been identified and discussed in recently published systematic reviews. ${ }^{10}$ Those barriers revolve around the interaction between families and the KMC intervention. They include buy-in and bonding (i.e. benefits of KMC to mothers and infants and perceptions of bonding between

Vol. 69, No. 11, November 2019 
mother and infant), social support (i.e. assistance from other people to perform $\mathrm{KMC}$ ), sufficient time to perform KMC, and medical concerns about mother or newborn health. Furthermore KMC adoption by caregivers within the context of the health system is associated with factors such as financing and service delivery. And within broad social context adherence to traditional newborn practices, stigma surrounding having a preterm infant, and gender roles regarding childcare are major barriers in KMC adoption. ${ }^{10,11}$

Pakistan can be a beneficiary of KMC through cheap and cost effective awareness programmes. The barriers and enablers identified in previous reviews and meta-analysis, if addressed in our settings, will help in KMC implementation. This article is necessary for generating a debate on why a cost effective community based intervention i.e., $\mathrm{KMC}$ is being over looked for much costlier options in already resource stricken settings. Outlining the hurdles faced during implementation of KMC in low socioeconomic conditions and identifying areas of opportunity for adopting KMC as part of health system in Pakistan are the keys for increasing compliance. The objective of this paper is to identify the opportunities and implications of KMC in resource constrained settings of rural Pakistan.

\section{Methods}

We did narrative review of available literature. ${ }^{12}$ The article does not intend to do a systematic review of all the available literature rather selected articles were reviewed for building this narrative. This commentary takes its roots in the public health perspective of infant mortality and its reduction. The very idea of this article arose as a consequence of journal club meeting at Aga Khan University that was about a systematic review regarding the barriers and enablers of KMC. ${ }^{11}$

For initial literature search of this article we used online databases i.e., Google Scholar, Science Direct, Ovid and PubMed. The Mesh terms used for online search were 'neonatal mortality', 'infant mortality', 'kangaroo mother care', 'kangaroo care', 'interventions to reduce neonatal mortality'. We reviewed articles that came up in each search to identify relevant articles. For relevant articles both researchers separately reviewed EndNote libraries which were merged after a consensus was achieved on them- this paper is not meant to be a systematic review hence PRISMA checklist was not applied. Following online database search we went through available data on WHO and United Nation's Children Fund (UNICEF) website to gather further information about KMC.

After data retrieval manuscript was synthesized by one researcher and then reviewed by the other and this required a series of annotations and changes made in the text.

\section{Results}

Following this iterative approach we constructed the results and discussion based upon identified literature relating to KMC barriers and enablers and finally recommendations for KMC implementation were developed.

Besides the benefit, KMC intervention has highlighted certain barriers and enablers. The barriers and enablers of KMC revolve around the care giver and family; finances and service delivery of health care and social barriers. We reviewed enablers and barriers separately in the context of Pakistan and have created following themes.

\section{Care giver and family}

Starting with the interaction between caregivers and the KMC intervention, certain factors such as buy-in and bonding, social support, time, and medical concerns have been major hindrances in KMC adoption. Hindrances for caregiver acceptance of KMC are focused around the mother. Factors that reduce maternal acceptance are related to healthcare worker's attitude; when healthcare workers do not clearly explain the benefits of KMC to mother, it results in lack of bonding by mothers with her preterm neonate. This lack of bonding is associated with inherent fear, stigma, shame, guilt or anxiety about having a preterm infant. 10 In some cases mothers did not want to keep the baby at all. ${ }^{13}$ Similarly barriers to parental buyin occur when caregivers perceive that their newborn does not enjoy KMC, hot climate made infants irritable during Skin to skin care (SSC). ${ }^{14}$

Finances and service delivery of health care lack of money for transportation and the distance to these hospital are often reported as the biggest challenges to KMC implementation in many studies. 15 This holds true for Pakistan where public transport is limited to larger cities and that too is not feasible for mothers. The waiting time for bus stop is very long and the congestion on roads and in the buses is a hindrance. Furthermore the cost of 
transport is also not feasible for low income countries such as Pakistan.

Subsidy and free medical service enabled parents to stay at the clinic longer as needed. ${ }^{16}$ Parents (as well as staff) noted that unlimited visitation hours enabled adoption of KMC. Health system concerns regarding financing travel, food, lodging, etc. may be partially alleviated by ensuring early discharge of mother and infant from the hospital (which should always be included as a component of KMC). Transportation and time costs may also be addressed by offering home visits by community health workers for infant follow up.

\section{Healthcare worker behaviour}

Patient satisfaction in maternal health services have direct link to healthcare worker behaviour which subsequently influences service utilization. ${ }^{17}$ Studies showed that mothers experienced a lack of support from healthcare workers and hospital staff as they were resistant to family participation in caring for the baby while in the hospital 18 and were loud and uncaring to parents. 19,20

Solution to this issue lies in proper training of health care workers, addressing the needs of health care workers to ensure that they are motivated. Quality assurance mechanisms such as introduction of feedback forms in the clinic, introduction of phone apps to rate staff attitude will help in introducing a check and balance mechanism in the facility. Furthermore pay raise for the workers if the patient feedback is good will incentivize KMC implementation and enhance its compliance in our setup.

\section{Social barriers}

In the broader social context demographics, economic and cultural factors influence the caregiver adoption of KMC. Surveys from 15 low income countries noted that health care professionals often found that KMC was thought of as substandard or as "the poor man's alternative". ${ }^{18}$ In rural settings traditional early bathing behaviour was seen as having numerous benefits and was identified as an ingrained behaviour by studies conducted in Ghana and Bangladesh. ${ }^{13}$ Divisions of labour and space by gender have been found to be barriers to male participation in newborn care, in general. ${ }^{19}$ Gender roles, the role of men in the household, and the roles of other family members also influenced KMC uptake. ${ }^{18}$ Solution to these issues must focus on efforts to implement and scale up $\mathrm{KMC}$ in a manner that ensure a positive experience for caregivers. Social support for assistance from other people to perform KMC is a major factor in KMC compliance as seen in literature. In most cases globally qualitative literature found that while practicing KMC parents did not feel supported by their families or communities. ${ }^{14}$ Many fathers felt that childcare should be the role of the mother and older generations, mothersin-law and grandmothers in particular, did not find KMC to be an appropriate method to care for newborns. 20 A solution to this issue was found when grandparents in the Agogo programme where church based mission hospital trained nearly 4000 grandparents and subsequently, grandparents provided individual and group counseling in their respective villages, using drama, song, and poems to share key messages. An evaluation of the programme concluded that grandparents were successful in promoting behaviour change surrounding maternal and newborn care. ${ }^{21}$ To address the reluctance of fathers to engage in childcare, fathers who have successfully engaged in SSC may become peer-mentors or demonstrators for other fathers and families. Previous reviews suggested that intervention name "Kangaroo Mother Care" might also be changed so that it does not directly imply the behaviour is performed only by the mother. ${ }^{11}$

\section{Discussion}

In the discussion section we have used these three areas for developing solutions in context of Pakistan. These factors are in context of literature on the barriers and enablers for adoption of KMC. ${ }^{10}$ Explaining in standardized manner to caregivers and their extended families, with attention paid to the concerns of mother and family, helps in increasing KMC compliance. The discussion about the effectiveness of KMC with parents could be done by caregivers who have successfully cared for a preterm or low birth weight baby in the past; such an approach is recommended in the Maternal and Child Health Integrated Programme (MCHIP) KMC Guide as well.22 Demonstrations of the right manner of KMC and supervised practice can enhance caregiver confidence. ${ }^{23}$

In an evaluation of a KMC inpatient ward of a tertiary hospital in Malawi, 10\% mothers whose children died reported the distance to the health facility or lack of transport money as the reason they did not go to the hospital when something was wrong with their newborn. Similarly, nearly $40 \%$ of mothers reported lack of transport 
money as the reason they did not go to the hospital for their follow up clinic appointments ${ }^{15,24}$ along with commuting between home and KMC wards. ${ }^{11}$

Previously done multi-country analysis of health system bottlenecks and potential solutions showed that community ownership and health financing bottlenecks were major issues for KMC implementation in both low and high mortality contexts, particularly in South Asia. Identified pathways to scale up KMC revolve globally around champion-led approach in which a small group lead by community person initiates and maintains the KMC intervention; another approach, the project-initiated approach focused on project driven and donor funded implementation of KMC and lastly the approach of health systems redesigning incorporated KMC in existing health system structure. Literature showed that the combination of all three pathways may lead to more rapid scale-up of KMC even in resource constrained settings, such as rural Pakistan. There are countries that have shifted from one to the next of these three approaches (e.g. Malawi) or have combined them (e.g. Rwanda). 25 Pakistan can also uses either of these three approaches or all of these three approaches and they can be customized according to our norms and culture.

At the community-level celebrations for the 'graduation' of a baby from KMC or discussions about KMC through radio or other public forums ${ }^{23}$ will foster in acceptance of $\mathrm{KMC}$ in society at large. Global integration of KMC into health system at large needs to focus on areas such as Sub-Saharan Africa and South East Asia. KMC adoption into culture of Pakistan where patriarchal norms are rife and high fertility rate results in involvement of mother to be divulged in between the newborn and previous children and hence becoming a barrier in KMC implementation should be addressed with community participation, involvement of social welfare and strengthening of the lady health workers programme.

Lack of maternal education will pose difficulty in implementing this intervention in Pakistan therefore a major effort needs to be put forth to not only increase the education and awareness of mothers but also teach $\mathrm{KMC}$ as an important health intervention in schools and colleges- using pictorial, audio/visual aids and props. The norms of gender identity, such as segregation in areas and functional domains in household where by men and women are accustomed to be involved in certain activities in the household will be a hindrance in implementation of KMC that can be overcome using community awareness and health promotion. Involvement of grandparents and religious figures will be beneficial as family plays a far greater role in health delivery compared to other nonAsian countries; the role of religion is embedded in the social fabric of Pakistan and hence will be a factor that if used properly will be beneficial in the longer run. Mosques and Friday sermons can be used to spread awareness for KMC.

Awareness campaigns using radio and television, using cricket and movie stars as brand ambassadors for the campaign to implement KMC will bear fruit and make it more acceptable in our setup. Creating conducive environment to implement $\mathrm{KMC}$ in relation to health system must focus on two areas, financial forces and service delivery. Financial forces will be the greatest driver of KMC in Pakistan and in that aspect community based approaches will be the key in driving down the cost of care and implementation of KMC. Service delivery improvement will be achieved through training of staff regarding $\mathrm{KMC}$, improving their communication skills using continued medical education short courses as a tool. Furthermore quality assurance checks placed in the health care setup such a patient satisfaction forms to be filled as compulsory requirement and present on the counter of clinics or in every bed/ room of hospital will ensure that constant feedback is taken from the patients and attendants resulting in change achieved in bottom up manner.

\section{Conclusion}

It is high time that the benefits of health care be utilized by rural population of the country at par with the urban population. This dream can only be fulfilled if the cost of health care was reduced in rural settings. KMC provides such an opportunity and hence it should be availed.

Disclaimer: None to declare.

Conflict of Interest: None to declare.

Funding disclosure: None to declare.

\section{References}

1. Wang $\mathrm{H}$, Liddell CA, Coates MM, Mooney MD, Levitz CE, Schumacher $A E$, et al. Global, regional, and national levels of neonatal, infant, and under-5 mortality during 1990-2013: a systematic analysis for the Global Burden of Disease Study 2013. Lancet 2014; 384: 957 79. 
2. Liu L, Johnson HL, Cousens S, Perin J, Scott S, Lawn JE, et al; Child Health Epidemiology Reference Group of WHO and UNICEF. Global, regional, and national causes of child mortality: an updated systematic analysis for 2010 with time trends since 2000. Lancet 2012; 379: 2151-61.

3. Bhutta ZA, Black RE. Global Maternal, Newborn, and Child Health - So Near and Yet So Far. N Engl J Med 2013; 369: 2226-35.

4. UNICEF. Levels and Trends in Child Mortality Report Geneva: WHO, World Bank, UN Population Division, 2014.

5. Studies NloP. Pakistan Demographic and Health Survey. Islamabad: National Institute of Population Studies, 2013

6. Khan A, Kinney MV, Hazir T, Hafeez A, Wall SN, Ali N, et al. Newborn survival in Pakistan: a decade of change and future implications. Health Policy Plann 2012; 27(suppl_3): iii72-iii87.

7. Nisar YB, Dibley MJ. Determinants of neonatal mortality in Pakistan: secondary analysis of Pakistan Demographic and Health Survey 2006-07. BMC Public Health 2014; 14: 663.

8. Chan GJ, Valsangkar B, Kajeepeta S, Boundy EO, Wall S. What is kangaroo mother care? Systematic review of the literature. J Glob Health 2016; 6: 010701.

9. WHO. WHO recommendations on interventions to improve preterm birth outcomes. Geneva: World Health Organization, 2015.

10. Chan GJ, Labar AS, Wall S, Atun R. Kangaroo mother care: a systematic review of barriers and enablers. Bull World Health Organ 2016; 94: 130-41J.

11. Smith ER, Bergelson I, Constantian S, Valsangkar B, Chan GJ. Barriers and enablers of health system adoption of kangaroo mother care: a systematic review of caregiver perspectives. BMC Pediatrics 2017; 17: 35 .

12. Liumbruno GM, Velati $C$, Pasqualetti $P$, Franchini $M$. How to write a scientific manuscript for publication. Blood Transfusion 2013; 11 : 217-26.

13. Waiswa P, Nyanzi S, Namusoko-Kalungi S, Peterson S, Tomson G, Pariyo GW. 'I never thought that this baby would survive; I thought that it would die any time': perceptions and care for preterm babies in eastern Uganda. Trop Med Int Health 2010;15: 1140-7.

14. Quasem I, Sloan NL, Chowdhury A, Ahmed S, Winikoff B, Chowdhury
AM. Adaptation of kangaroo mother care for community-based application. J Perinatol 2003; 23: 646-51.

15. Blencowe $H$, Kerac $M$, Molyneux E. Safety, effectiveness and barriers to follow-up using an 'early discharge' Kangaroo Care policy in a resource poor setting. J Trop Pediatr 2009; 55: 244-8.

16. Kambarami RA, Chidede O, Pereira N. Long-term outcome of preterm infants discharged home on kangaroo care in a developing country. Ann Trop Paediatr 2003; 23: 55-9.

17. Kujawski S, Mbaruku G, Freedman LP, Ramsey K, Moyo W, Kruk ME. Association Between Disrespect and Abuse During Childbirth and Women's Confidence in Health Facilities in Tanzania. Matern child Health J 2015; 19: 2243-50.

18. Charpak N, Ruiz-Pelaez JG. Resistance to implementing Kangaroo Mother Care in developing countries, and proposed solutions. Acta Paediatr 2006; 95: 529-34.

19. Dumbaugh M, Tawiah-Agyemang C, Manu A, ten Asbroek GH Kirkwood B, Hill Z. Perceptions of, attitudes towards and barriers to male involvement in newborn care in rural Ghana, West Africa: a qualitative analysis. BMC Pregnancy Childbirth 2014; 14: 269.

20. Kambarami R. Kangaroo care and multiple births. Ann Trop Paediatr 2002; 22: 107-8.

21. Zimba E, Kinney MV, Kachale F, Waltensperger KZ, Blencowe $\mathrm{H}_{\text {, }}$ Colbourn T, et al. Newborn survival in Malawi: a decade of change and future implications. Health Policy Plan 2012; 27(suppl_3): iii88iii103.

22. MCHIP. Kangaroo Mother Care: Implementation Guide. USAID 2012.

23. Kumar V, Mohanty S, Kumar A, Misra RP, Santosham M, Awasthi S et al. Effect of community-based behaviour change management on neonatal mortality in Shivgarh, Uttar Pradesh, India: a clusterrandomised controlled trial. Lancet 2008; 372: 1151-62.

24. Boo NY, Jamli FM. Short duration of skin-to-skin contact: effects on growth and breastfeeding. J Paediatr Child Health 2007; 43: 831-6.

25. Vesel L, Bergh AM, Kerber KJ, Valsangkar B, Mazia G, Moxon SG, et al. Kangaroo mother care: a multi-country analysis of health system bottlenecks and potential solutions. BMC Pregnancy Childbirth 2015; 15: S5. 